\title{
Study on the Number of Calculations and Number of Blinks when Performing Uchida-Kraepelin Psychodiagnostic Test
}

\author{
Shunsuke Nakano, Yoichi Kageyama* \\ Akita University, 1-1, Tegata Gakuen-machi, Akita-shi, Akita 010-8502, Japan \\ *Corresponding Author: kageyama@ie.akita-u.ac.jp
}

\begin{abstract}
Evaluating physical and mental conditions automatically and quantitatively contributes to the improvement of quality of life. The Uchida-Kraepelin psychodiagnostic test not only estimates the character of a person but also reflects his/her mental and physical conditions. To associate the results of the Uchida-Kraepelin psychodiagnostic test reflecting mental and physical conditions with objective data, the relation between the number of blinks and number of calculations was investigated. The results obtained via experiments were as follows: (a) There was a negative correlation between the number of calculations and number of blinks. (b) The "late increase rate" indicated by the Uchida-Kraepelin psychodiagnostic test was also reflected in the test on a personal computer. (c) There was a significant difference between the first and second periods in the number of blinks. (d) It was effective to exclude outliers to detect more significant differences between the first and second periods.
\end{abstract}

Keywords: quality of life, blink, Uchida-Kraepelin psychodiagnostic test.

\section{Introduction}

It is essential to improve the quality of life (QOL) of each individual in a super-aging society ${ }^{(1)}$. However, subjective evaluation is often used as a typical method to evaluate $\mathrm{QOL}^{(2)}$, and it is difficult to estimate unconscious mental and physical conditions. Under conditions of stress such as exercise and drinking, the tendency of the results obtained using the Uchida-Kraepelin psychodiagnostic test is expected to change ${ }^{(3)}$. Clarifying the relation between the change in tendency owing to stress when performing the Uchida-Kraepelin psychodiagnostic test and the features obtained from the image contributes to an objective estimate of QOL. Therefore, in this study, we focus on the blink as a feature quantity, and examine the relation between the number of blinks and the Uchida-Kraepelin psychodiagnostic test results.

\section{Data Used}

\subsection{Uchida-Kraepelin Psychodiagnostic Test}

Refer to the prepared number sequence, and add the two adjacent numbers. If the result is a single-digit number, write that number; if it is a two-digit number, write the last digit. After performing this task for 15 min continuously, rest for $5 \mathrm{~min}$, and perform the same task for $15 \mathrm{~min}$ continuously again. The transition of the value obtained by adding the number of calculations every minute is used as a feature. In this study, the face image of the subject is acquired from the front. Therefore, the Uchida-Kraepelin psychodiagnostic test was performed using the program running on a personal computer (PC). Fig. 1 shows an example of the program execution screen. The answer and correction of the calculation are performed through mouse operation, and the switching of the problem is performed through the keyboard operation. For the answers corrected by the subjects, it was

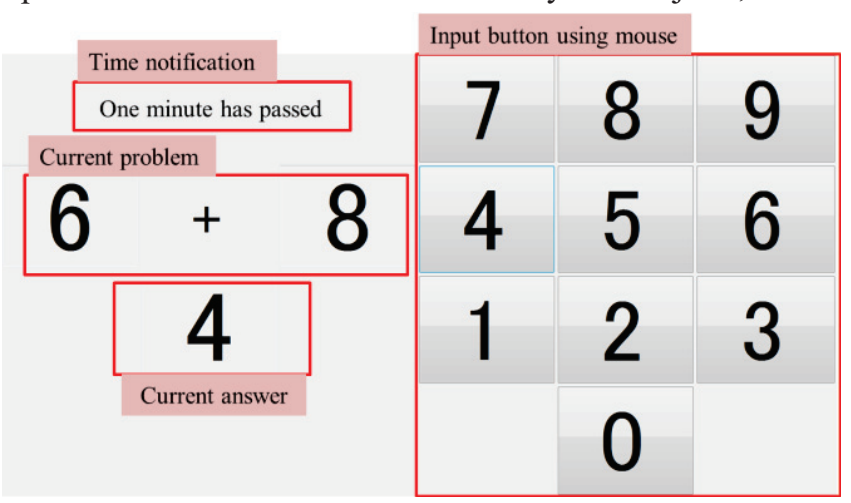

Fig. 1. Example of program execution screen. 


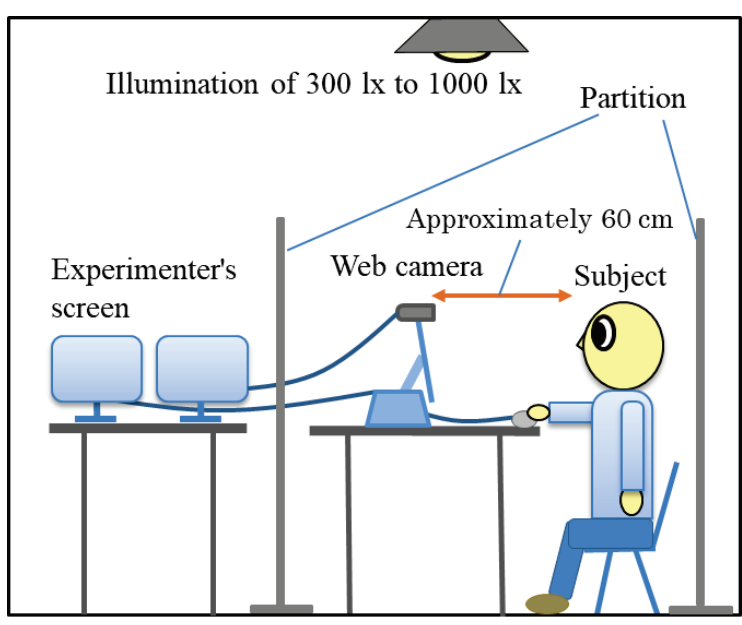

Fig. 2. Experiment environment.

judged that they were correct. The number of calculations is calculated except for the solution judged to be incorrect.

\subsection{Data Acquisition Experiment}

There were five subjects (A to E, men in their twenties), and experiments for acquiring moving image data were conducted four times on different days per person (Subject D left the experiment on day 3 owing to poor health). Photographing was performed using a web camera at an illumination of $300 \mathrm{~lx}$ to $1000 \mathrm{~lx}$. The distance between the subject and the web camera is approximately $60 \mathrm{~cm}$. Fig. 2 shows the experiment environment. The face image was taken from the front at the same time as the subject was undergoing the Uchida-Kraepelin psychodiagnostic test on the PC. Furthermore, a questionnaire survey was administered before and after the experiment to record the subject's mental and physical conditions. The data used in this study were acquired in accordance with the ethical regulations concerning studies involving humans at Akita University, Japan.

\subsection{Questionnaire}

Whether a subject receives stress depends on his ability at calculation, troublesome experiments, and conditions of the day. Work efficiency and calculation speed change owing to stress. Therefore, to consider the difference in the values for each subject, a questionnaire survey was administered to record the subject's mental and physical conditions. Furthermore, the stress received by the subject under the experiments was investigated. An example of the survey items of the questionnaire is shown below.

- Physical conditions (nausea, sweating, etc.)

- Troublesome calculation

- Troublesome PC operation
- Screen readability

- Stress caused by photography

- Psychological burden in recent time

- Drinking on the previous day

- Working part-time on the previous day

- Bedtime and wake up time

- Exercise habits

- Breakfast habits

- Vision correction (glasses, contact lenses)

\section{Analytical Method}

\subsection{Blink Frame Setting}

In this study, the number of blinks measured manually was used as the basic data for analysis. The visible image data acquired through the experiment were visually confirmed frame by frame. Fig. 3 shows an example of blink frame setting. The most closed frame was set as the blink frame while the eyelids opened and closed. After manually recording blink frames, the number of blink frames per minute was calculated as the measured value of the number of blinks per minute.

\subsection{Calculation of Correlation Coefficient}

Using the calculation answers recorded on the PC, the number of calculations per minute was calculated. Fig. 4 shows a transition example of the number of calculations and the number of blinks. Fig. 5 shows a correlation diagram example of the number of calculations and the number of blinks. In addition, assuming that there is a negative correlation between the number of calculated answers and the number of blinks, the correlation coefficient between the data was calculated.

\subsection{Late Increase Rate}

According to the Uchida-Kraepelin psychodiagnostic test, the late increase rate in the standard case tends to increase. However, if the subject finds it difficult to concentrate owing to his personality, or if the mental

\section{Blink frame}
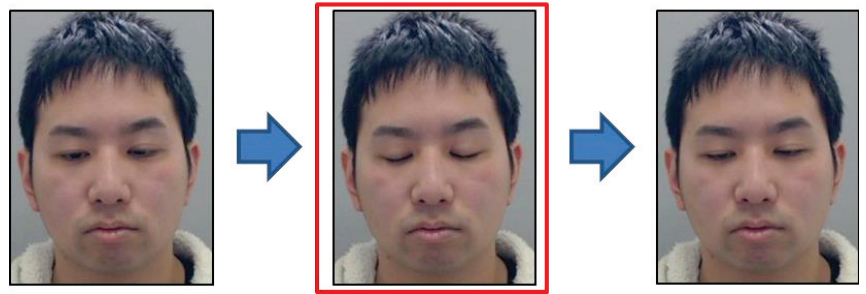

Fig. 3. Example of blink frame setting. 


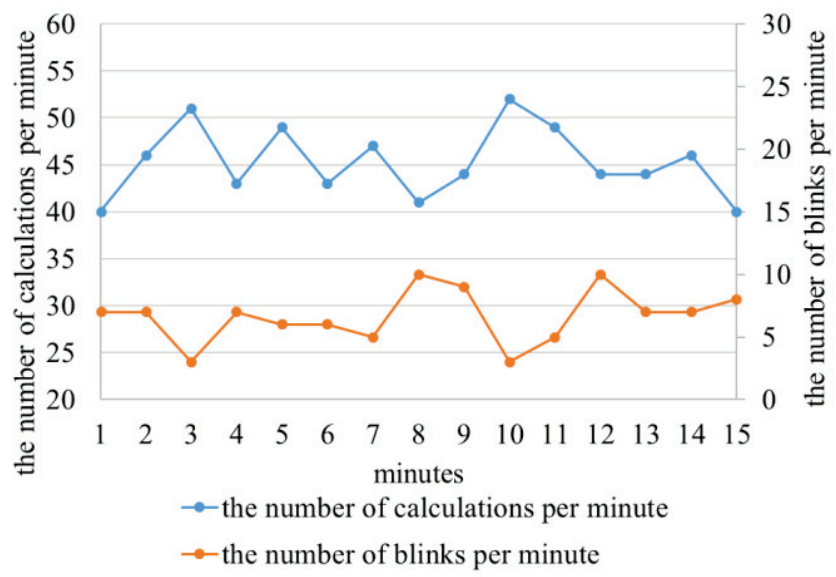

Fig. 4. Transition example of the number of calculations and the number of blinks.

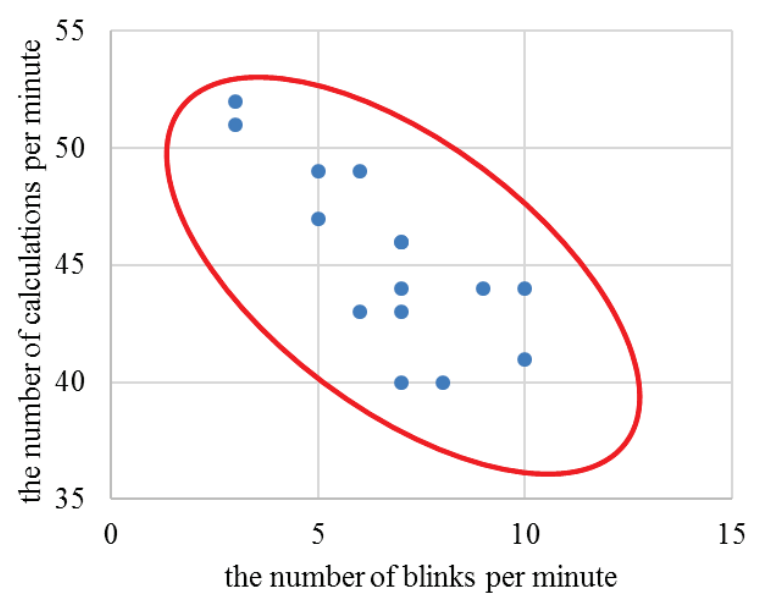

Fig. 5. Correlation diagram example of the number of calculations and the number of blinks.

conditions or physical conditions at the time of the test are not appropriate, the late increase rate declines ${ }^{(4)}$. In this study, to investigate whether the same results can be obtained in the reproduction of the Uchida-Kraepelin psychodiagnostic test on the PC, the corresponding t-test ${ }^{(5)}$ was applied to the number of calculations in the first and second periods, and the same examination was conducted to investigate whether the number of blinks differs between the first and second periods.

\subsection{Examination Excluding Outliers}

The number of blinks and the number of calculations may have a large variance owing to the influence of some values. Therefore, the t-test was conducted again after excluding the outliers in the data. The outliers were set based on the quartile deviation. Sometimes, the number of elements in the first and second periods did not match owing to the exclusion of the outliers. Thus, Welch's test ${ }^{(5)}$ was used.

\section{Results}

\subsection{Calculation Results of Correlation Coefficient}

Table 1 shows the correlation coefficient between the number of calculations and the number of blinks. The correlation coefficient is approximately -0.40 or less, indicating negative correlation. The results suggest that it is possible to estimate the transition in the number of calculations by focusing on the transition in the number of blinks.

\subsection{Results of Late Increase Rate}

Table 2 shows the results of t-test on the number of calculations. Table 3 shows the results of t-test on the number of blinks. A significant difference was observed in seven data points in the number of calculations and two data points in

Table 1. Correlation coefficient between the number of calculations and the number of blinks.

\begin{tabular}{|c|c||r|r|r|r|r|}
\hline \multicolumn{2}{|c||}{} & \multicolumn{6}{c|}{ Subject } \\
\cline { 2 - 7 } \multicolumn{2}{|c|}{ D } & \multicolumn{1}{c|}{ B } & \multicolumn{1}{c|}{ C } & \multicolumn{1}{c|}{ D } & \multicolumn{1}{c|}{ E } \\
\hline \hline \multirow{2}{*}{ Day 1} & First & -0.52 & -0.06 & 0.44 & -0.55 & -0.27 \\
\cline { 2 - 7 } & Second & -0.37 & -0.16 & 0.24 & -0.42 & -0.39 \\
\hline \multirow{2}{*}{ Day 2 } & First & -0.57 & -0.47 & -0.08 & -0.43 & -0.47 \\
\cline { 2 - 7 } & Second & -0.65 & -0.63 & -0.48 & -0.35 & -0.63 \\
\hline \multirow{2}{*}{ Day 3 } & First & -0.77 & -0.47 & 0.09 & & -0.51 \\
\cline { 2 - 7 } & Second & -0.47 & -0.44 & -0.40 & & -0.61 \\
\hline \multirow{2}{*}{ Day 4 } & First & -0.70 & -0.55 & -0.19 & -0.49 & 0.15 \\
\cline { 2 - 7 } & Second & -0.70 & -0.53 & 0.15 & -0.04 & 0.19 \\
\hline
\end{tabular}

Table 2. Results of t-test on the number of calculations.

\begin{tabular}{|c|c|c|c|c|c|}
\hline Subject & Day & First ave. & Second ave. & Difference & p-value \\
\hline \multirow{4}{*}{ A } & 1 & 41.1 & 44.1 & -2.9 & 0.058 \\
\hline & 2 & 42.1 & 44.1 & -2.1 & 0.228 \\
\hline & 3 & 45.3 & 44.6 & 0.7 & 0.611 \\
\hline & 4 & 44.5 & 45.1 & -0.6 & 0.698 \\
\hline \multirow{4}{*}{ B } & 1 & 43.3 & 47.7 & -4.3 & 0.021 \\
\hline & 2 & 48.7 & 46.8 & 1.9 & 0.029 \\
\hline & 3 & 48.1 & 50.7 & -2.7 & 0.098 \\
\hline & 4 & 52.9 & 54.9 & -1.9 & 0.770 \\
\hline \multirow{4}{*}{$\mathrm{C}$} & 1 & 52.1 & 54.3 & -2.3 & 0.005 \\
\hline & 2 & 51.3 & 53.0 & -1.7 & 0.190 \\
\hline & 3 & 53.8 & 54.0 & -0.2 & 0.001 \\
\hline & 4 & 53.3 & 56.1 & -2.7 & 0.056 \\
\hline \multirow{4}{*}{ D } & 1 & 49.0 & 50.0 & -1.0 & 0.377 \\
\hline & 2 & 50.3 & 52.7 & -2.4 & 0.027 \\
\hline & 3 & & & & \\
\hline & 4 & 54.2 & 55.7 & -1.5 & 0.573 \\
\hline \multirow{4}{*}{$\mathrm{E}$} & 1 & 49.7 & 43.3 & 6.4 & 1.000 \\
\hline & 2 & 51.0 & 50.3 & 0.7 & 0.001 \\
\hline & 3 & 48.7 & 48.7 & 0.0 & 0.160 \\
\hline & 4 & 54.3 & 55.3 & -1.1 & 0.019 \\
\hline
\end{tabular}


Table 3. Results of t-test on the number of blinks.

\begin{tabular}{|c|c|c|c|c|c|}
\hline Subject & Day & First ave. & Second ave. & Difference & p-value \\
\hline \multirow{4}{*}{ A } & 1 & 7.1 & 7.1 & 0.1 & 0.945 \\
\hline & 2 & 8.2 & 8.3 & -0.1 & 0.962 \\
\hline & 3 & 6.7 & 7.7 & -1.1 & 0.100 \\
\hline & 4 & 8.7 & 7.4 & 1.3 & 0.236 \\
\hline \multirow{4}{*}{ B } & 1 & 23.7 & 19.2 & 4.5 & 0.347 \\
\hline & 2 & 11.1 & 14.5 & -3.4 & 0.060 \\
\hline & 3 & 17.6 & 14.3 & 3.3 & 0.833 \\
\hline & 4 & 18.4 & 18.7 & -0.3 & 0.031 \\
\hline \multirow{4}{*}{ C } & 1 & 7.2 & 5.4 & 1.8 & 0.810 \\
\hline & 2 & 7.7 & 7.4 & 0.3 & 0.090 \\
\hline & 3 & 10.1 & 7.2 & 2.9 & 0.001 \\
\hline & 4 & 8.5 & 8.3 & 0.3 & 0.317 \\
\hline \multirow{4}{*}{ D } & 1 & 2.4 & 2.9 & -0.5 & 0.390 \\
\hline & 2 & 3.0 & 2.2 & 0.8 & 0.171 \\
\hline & 3 & & & & 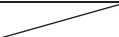 \\
\hline & 4 & 2.9 & 2.1 & 0.8 & 0.705 \\
\hline \multirow{4}{*}{ E } & 1 & 8.2 & 26.9 & -18.7 & 0.387 \\
\hline & 2 & 9.5 & 8.7 & 0.7 & 0.133 \\
\hline & 3 & 15.8 & 12.7 & 3.1 & 0.145 \\
\hline & 4 & 22.7 & 21.3 & 1.3 & 0.963 \\
\hline
\end{tabular}

Table 4. Results of Welch's test on the number of calculations after excluding outliers.

\begin{tabular}{|c|c|c|c|c|c|}
\hline Subject & Day & First ave. & Second ave. & Difference & $\mathrm{p}$-value \\
\hline \multirow{4}{*}{ A } & 1 & 42.0 & 43.3 & -1.3 & 0.261 \\
\hline & 2 & 42.5 & 42.5 & -0.1 & 0.932 \\
\hline & 3 & 43.6 & 44.6 & -1.0 & 0.324 \\
\hline & 4 & 44.5 & 44.8 & -0.3 & 0.840 \\
\hline \multirow{4}{*}{ B } & 1 & 42.9 & 48.3 & -5.4 & 0.001 \\
\hline & 2 & 48.7 & 46.3 & 2.4 & 0.036 \\
\hline & 3 & 48.1 & 50.7 & -2.7 & 0.016 \\
\hline & 4 & 53.8 & 54.8 & -1.0 & 0.154 \\
\hline \multirow{4}{*}{ C } & 1 & 51.8 & 55.0 & -3.3 & 0.001 \\
\hline & 2 & 51.6 & 53.1 & -1.5 & 0.023 \\
\hline & 3 & 53.8 & 54.1 & -0.3 & 0.600 \\
\hline & 4 & 53.8 & 55.5 & -1.8 & 0.003 \\
\hline \multirow{4}{*}{ D } & 1 & 46.8 & 48.5 & -1.7 & 0.017 \\
\hline & 2 & 50.5 & 52.0 & -1.5 & 0.084 \\
\hline & 3 & & & & 7 \\
\hline & 4 & 53.8 & 55.3 & -1.5 & 0.055 \\
\hline \multirow{4}{*}{ E } & 1 & 49.2 & 43.3 & 5.9 & 0.001 \\
\hline & 2 & 51.5 & 50.6 & 0.9 & 0.122 \\
\hline & 3 & 48.7 & 47.6 & 1.1 & 0.285 \\
\hline & 4 & $\begin{array}{l}54.3 \\
\end{array}$ & 55.8 & -1.5 & 0.030 \\
\hline
\end{tabular}

the number of blinks. The results suggest that the late increase rate in the Uchida-Kraepelin psychodiagnostic test is also reflected in the test on the PC. In addition, there is a peculiar change in the late increase rate in the number of blinks.

\subsection{Results of Examination Excluding Outliers}

Table 4 shows the results of Welch's test on the number of calculations after excluding outliers. Table 5 shows the results of Welch's test on the number of blinks after
Table 5. Results of Welch's test on the number of blinks after excluding outliers.

\begin{tabular}{|r|r|r|r|r|r|}
\hline Subject & Day & First ave. & Second ave. & Difference & p-value \\
\hline \hline & 1 & 6.5 & 6.7 & -0.2 & 0.769 \\
\cline { 2 - 6 } A & 2 & 7.5 & 8.3 & -0.8 & 0.370 \\
\cline { 2 - 6 } & 3 & 6.7 & 6.4 & 0.4 & 0.508 \\
\cline { 2 - 6 } & 4 & 7.6 & 6.7 & 0.9 & 0.268 \\
\hline \multirow{4}{*}{ B } & 1 & 20.8 & 17.1 & 3.7 & 0.037 \\
\cline { 2 - 6 } & 2 & 8.3 & 12.5 & -4.2 & 0.042 \\
\cline { 2 - 6 } & 3 & 12.2 & 14.3 & -2.1 & 0.389 \\
\cline { 2 - 6 } & 4 & 12.3 & 11.6 & 0.7 & 0.822 \\
\hline \multirow{4}{*}{ C } & 1 & 6.9 & 5.4 & 1.5 & 0.124 \\
\cline { 2 - 6 } & 2 & 6.9 & 6.8 & 0.2 & 0.834 \\
\cline { 2 - 6 } & 3 & 8.6 & 6.4 & 2.2 & 0.011 \\
\hline \multirow{4}{*}{ D } & 4 & 7.9 & 6.0 & 1.9 & 0.055 \\
\cline { 2 - 6 } & 1 & 2.0 & 2.6 & -0.6 & 0.083 \\
\cline { 2 - 6 } & 2 & 2.5 & 1.7 & 0.8 & 0.085 \\
\cline { 2 - 6 } & 3 & & & & \\
\hline \multirow{3}{*}{ E } & 1 & 2.8 & 1.6 & 1.2 & 0.002 \\
\cline { 2 - 6 } & 2 & 7.7 & 28.5 & -20.8 & 0.001 \\
\cline { 2 - 6 } & 3 & 10.9 & 11.7 & -0.8 & 0.586 \\
\cline { 2 - 6 } & 4 & 22.1 & 20.8 & 1.3 & 0.331 \\
\hline
\end{tabular}

excluding outliers. A significant difference was observed in nine data points in the number of calculations and five data points in the number of blinks. The results suggest that it is useful to exclude outliers when calculating the late increase rate using the number of calculations and the number of blinks.

\section{Discussion}

The number of calculations and the number of blinks generally tended to show a negative correlation, but weak correlation, no correlation, and positive correlation were also obtained. In particular, the latter tendencies were frequently observed in the first-day data. In data acquisition experiments, there are many unfamiliar elements such as the first operation, environment, and problems. In addition, the level of proficiency in work increases with the number of times the work is performed, and the number of calculations also significantly increases ${ }^{(3,6)}$. These facts indicate that the data on the first day of the experiment reflected the internal disturbance caused by the subject's inexperience in the experiments, showing a tendency different from the whole.

\section{Conclusion}

The purpose of this study is to establish a method to objectively estimate QOL. The tendency of the number of calculations and the number of blinks when performing the 
Uchida-Kraepelin psychodiagnostic test on a PC was examined. Consequently, the number of calculations and the number of blinks tended to show a negative correlation. Moreover, the late increase rate specific to the UchidaKraepelin psychodiagnostic test showed that it might be reflected in the test method using the PC. Furthermore, not only the number of calculations but also the number of blinks may have a significant difference between the average values of the first and second periods, indicating the possibility of having a distinctive feature such as the late increase rate. In the future, we plan to construct a system to measure the frequency of blinks automatically.

\section{Acknowledgment}

This study has been supported by a JSPS KAKENHI Grant No. JP19K12909.

\section{References}

(1) Ministry of Internal Affairs and Communications : "Special issue: How to bring energy and growth to Japan by strategic use of smart ICT", http:/www. soumu.go.jp/johotsusintokei/whitepaper/ja/h25/html/nc 123110.html (Accessed 27 May, 2019)

(2) Koujiro Shimozuma : "History and Future Perspective of QOL Assessment Research", Japanese journal of behavioral medicine, Vol. 21, No. 1, pp. 4-7, 2015

(3) Junichi Kurokawa : "Evaluation by Repeated Use of Uchida-Kraepelin Psychodiagnostic Test (1st Report) : Evaluation of Various Conditions by Daily Use of the Test", Japanese journal of occupational medicine and traumatology, Vol. 60, No. 2, pp. 74-90, 2012

(4) Tsuchiya Bookstore Editorial Department : "Job aptitude test Uchida-Kraepelin psychodiagnostic test Complete understanding manual", Tokyo, Japan, Tsuchiya bookstore Corporation, 2008

(5) Shinichi Kurihara : "Introductory statistics from testing to multivariate analysis design", Tokyo, Japan, Ohmsha, 2011

(6) Yasuaki Fukuda and Ryuji Sakai : "A Method of Work Appraisement by Professing Equation in Production", Journal of Japan Industrial Management Association, Japan Industrial Management Association, Vol. 32, No. 3, pp. 188-194, 1981 